\title{
Monte Carlo Study of Doping Change and Disorder Effect on Double Exchange Ferromagnetism
}

\author{
Yukitoshi Motome \\ RIKEN (The Institute of Physical and Chemical Research), \\ 2-1 Hirosawa, Wako, Saitama 351-0198, Japan \\ Nobuo Furukawa \\ Department of Physics, Aoyama Gakuin University, \\ 5-10-1 Fuchinobe, Sagamihara, Kanagawa 229-8558, Japan
}

(Dated: November 1, 2018)

\begin{abstract}
Phase diagram and critical properties are studied for three-dimensional double exchange model with and without quenched disorder. Employing the Monte Carlo method and the systematic analysis on the finite-size effect, we estimate the Curie temperature and the critical exponent as functions of the doping concentration and the strength of the random potential. The Curie temperature well scales to the kinetic energy of electrons in the ground state as expected for this kinetics-driven ferromagnetism. The universality class of this transition is described by the short-range Heisenberg fixed point. The results are compared with the experimental results in the colossal magnetoresistance manganites.

PACS numbers: 75.40.Cx, 75.47.Lx, 71.10.-w
\end{abstract}

\section{INTRODUCTION}

Since Zener's pioneering work, $\frac{1}{\underline{-1}}$ the double exchange (DE) model and its extensions have been studied to understand the variety of magnetic and transport properties including the colossal magnetoresistance (CMR) in perovskite manganese oxides 2.3 .4 .5 The original DE model, which contains the single-band electrons interacting with the localized spins through the Hund's-rule coupling (the Hamiltonian will be explicitly given in Sec. IA , qualitatively explains the stability of the ferromagnetic metallic state and the negative magnetoresistance. There, the parallel configuration of localized spins leads to the kinetic energy gain of electrons, and vice versa. The effective ferromagnetic interaction between localized spins is mediated by the motion of electrons, and is called the DE interaction. Despite of the long survey for more than half century, however, the quantitative aspects of the thermodynamics of this model have not been fully explored thus far. This is mainly because the system is in the strongly-correlated regime in the sense that the Hund's-rule coupling is much larger than the bandwidth of electrons. Both thermal and spatial fluctuations are crucial in the thermodynamics of this itinerant electron system, and they are difficult to handle in a controllable manner.

Monte Carlo (MC) calculation is one of the powerful tools to treat such strong correlations properly. In the present problem, since the wavefunction of itinerant electrons is extended, it is crucial to make a systematic finite-size scaling analysis for obtaining a reliable result in the thermodynamic limit. Recently, there have been some developments in the MC algorithm which enable us to handle larger size systems than ever in a reasonable computational time ${ }^{6.7 .8}$ The importance of spatial fluctuations and finite-size effects has been ex- amined by comparing the MC results with the meanfield results or the dynamical mean-field approximation (DMFA) results $\frac{9}{-1}$ The reliable estimate of the Curie temperature $T_{\mathrm{C}}$ has been obtained by the systematic scaling analysis ${ }^{8,9.10}$ The critical exponents have been also estimated, and it is shown that the exponents are consistent with those of the Heisenberg spin model with short-range interactions 8.9 .10 .11 .12

These previous MC calculations have been mainly performed at the doping concentration $x=0.5$ ( 0.5 electrons per site on average), where the kinetic energy of electrons is maximum, namely, $T_{\mathrm{C}}$ becomes the highest. In real compounds, for instance, in $\mathrm{La}_{1-x} \mathrm{Sr}_{x} \mathrm{MnO}_{3}$, the ferromagnetic metallic phase is stabilized at $0.15 \lesssim x \lesssim 0.6$, and $T_{\mathrm{C}}$ becomes maximum at $x \simeq 0.3$ and slightly reduces for $x>0.3{ }^{13}$ This might be due to an instability toward the A-type antiferromagnetic state or the chargeordered state near $x=0.5,14.15,16$ These instabilities are beyond the simple DE model. The $x$ dependence of $T_{\mathrm{C}}$ for $0.1 \lesssim x \lesssim 0.3$ in $\mathrm{La}_{1-x} \mathrm{Sr}_{x} \mathrm{MnO}_{3}$ has been favorably compared with the DMFA results 17 However, the recent MC study has revealed the insufficiency of DMFA and the importance of spatial fluctuations as mentioned above. One of the purposes of this work is to determine $T_{\mathrm{C}}$ for wide regions of $x$ by applying the advanced $\mathrm{MC}$ technique and to clarify the phase diagram of the DE model. We will compare the numerical results with the experimental results quantitatively.

Another purpose of this work is to clarify the disorder effect on the critical properties of the ferromagnetic transition in the DE model. Disorder suppresses the kinetic energy of electrons and reduces $T_{\mathrm{C}}$. Although this reduction has been also studied by DMFA $18,19,20,21$ and the MC calculation for small size clusters, ${ }^{22}$ here we give more precise estimates employing the advanced MC method and the systematic finite-size scaling. Through 
the quantitative comparison between $T_{\mathrm{C}}$ and the kinetic energy of electrons, we examine how the kinetics governs the DE ferromagnetism in the disordered case. We also estimate the critical exponent to clarify the universality class in the disordered case. These are also motivated by the experiments on the $A$-site substitution in $A \mathrm{MnO}_{3}$ at a fixed valence $x$ which indicate the relevance of the chemical disorder of the $A$-site ions ${ }^{23.24 .25 .26}$ We will make a quantitative comparison between theory and experiment.

This paper is organized as follows. In Sec. [II we introduce the DE model including the random potential. The MC method as well as the details of numerical conditions is also described here. In Sec. III] we show the numerical results. $T_{\mathrm{C}}$ and the critical exponent are estimated by the systematic finite-size scaling in both cases with and without the random potential. We discuss the numerical results in comparison with the experimental results in CMR manganites in Sec. IV Section $\nabla$ is devoted to summary.

\section{MODEL AND METHOD}

\section{A. Model}

The DE model considered here consists of itinerant electrons in the single band which interact with localized spins at each site through the Hund's-rule coupling. The Hamiltonian is given in the form 1

$$
\mathcal{H}_{\mathrm{DE}}=-\sum_{\langle i j\rangle \sigma} t\left(c_{i \sigma}^{\dagger} c_{j \sigma}+\text { h.c. }\right)-J_{\mathrm{H}} \sum_{i} \boldsymbol{\sigma}_{i} \cdot \boldsymbol{S}_{i},
$$

where the first term describes the electron hopping between the nearest-neighbor sites and the second term represents the Hund's-rule coupling between the Pauli spin $\boldsymbol{\sigma}$ of electrons and the localized spin $\boldsymbol{S}$ (the coupling is ferromagnetic, namely, $J_{\mathrm{H}}$ is positive). Additionally, we take account of the the random on-site potential as

$$
\mathcal{H}_{\varepsilon}=\sum_{i \sigma} \varepsilon_{i} c_{i \sigma}^{\dagger} c_{i \sigma}
$$

where we consider the binary distribution, namely, $\varepsilon_{i}$ takes the value of $\pm \Delta$ with equal probability randomly in each site. The total Hamiltonian is given by $\mathcal{H}=$ $\mathcal{H}_{\mathrm{DE}}+\mathcal{H}_{\varepsilon}$.

In the following, for simplicity, we consider the limit of $J_{\mathrm{H}} \rightarrow \infty$. In this limit, the electron spin $\boldsymbol{\sigma}$ is completely parallel to the localized spin $\boldsymbol{S}$ in each site, and states with $\boldsymbol{\sigma}$ antiparallel to $\boldsymbol{S}$ are projected out. This simplifies the model to the effective spinless-fermion model in the form ${ }^{2}$

$$
\mathcal{H}=-\sum_{\langle i j\rangle} \tilde{t}_{i j}\left(\tilde{c}_{i}^{\dagger} \tilde{c}_{j}+\text { h.c. }\right)+\sum_{i} \varepsilon_{i} \tilde{c}_{i}^{\dagger} \tilde{c}_{i}
$$

where the transfer integral $\tilde{t}_{i j}$ depends on the relative angle of localized spins at $i$ and $j$ th site as

$$
\tilde{t}_{i j}=t\left(\cos \frac{\theta_{i}}{2} \cos \frac{\theta_{j}}{2}+\sin \frac{\theta_{i}}{2} \sin \frac{\theta_{j}}{2} \mathrm{e}^{-\mathrm{i}\left(\phi_{i}-\phi_{j}\right)}\right) .
$$

Here, the angles $\theta_{i}$ and $\phi_{i}$ are defined as $S_{i}^{x}=$ $S \sin \theta_{i} \cos \phi_{i}, S_{i}^{y}=S \sin \theta_{i} \sin \phi_{i}$, and $S_{i}^{z}=S \cos \theta_{i}$, where $S=\left|\boldsymbol{S}_{i}\right|$ is the magnitude of the localized spin. Thus, the transfer integral becomes a complex variable whose amplitude is proportional to $\cos \left(\theta_{i}-\theta_{j}\right) / 2$ and phase is governed by the so-called Berry phase $\exp \left\{-\mathrm{i}\left(\phi_{i}-\phi_{j}\right)\right\}$.

One more simplification we introduce here is to treat the localized spins in the classical limit of $S \rightarrow \infty$. Then, the present model (3) describes the strong interplay between the quantum itinerant electrons and the classical localized spins. If the configuration of the localized spins $\left\{\boldsymbol{S}_{i}\right\}$ is frozen, the problem is simply the free electrons in the random magnetic field. However, in the present problem, the localized spins are not the fixed external magnetic field but internal degrees of freedom of the system. The configurations of $\left\{\boldsymbol{S}_{i}\right\}$ are determined thermodynamically through the interaction with itinerant electrons. Namely, thermal equilibrium is achieved to optimize $\left\{\boldsymbol{S}_{i}\right\}$ which reconciles the kinetics of electrons and the entropy of the localized spins.

\section{B. Method and Numerical conditions}

In the following, we calculate the critical properties of the ferromagnetic transition in model (31) defined on the three-dimensional cubic lattice. We employ the truncated polynomial-expansion MC (TPEMC) method which is recently developed by the authors ${ }^{?}$ This technique is based on the polynomial-expansion MC method,,$\frac{6}{}$ and has advantage in the computational cost by introducing effective truncations in the polynomial expansion. Readers are referred to Ref. 7 for the details of the algorithm. Using this method, we calculate the physical quantities in the finite-size clusters of $N=L^{3}$ from $L=6$ to 16 . Systematic analyses on the finite-size corrections are performed for those series.

We typically perform 10000 MC samplings for measurements after 1000 steps for thermalization in the absence of the disorder. The results are divided into five bins to estimate the statistical errors by the variance among the bins. In the presence of the disorder, for a given configuration of $\left\{\varepsilon_{i}\right\}$, we typically perform 1000 MC samplings for measurements after 1000 steps for thermalization. We repeat this for typically 16 different configurations to estimate the errors by taking the random average among the results for each $\left\{\varepsilon_{i}\right\}$. We take the half bandwidth $W=6 t$ at $J_{\mathrm{H}}=\Delta=0$ as an energy unit.

We choose twisted boundary conditions to reduce the ground state degeneracy in the finite-size systems 27 by introducing the magnetic flux $\Phi=\pi / 4, \pi / 2$, and $3 \pi / 4$ in the $x, y$, and $z$ direction, respectively. The magnetic flux $\Phi$ is included by the so-called Peierls factor in the phase of the transfer integrals as $\tilde{t}_{i j} \rightarrow \tilde{t}_{i j} \exp (\mathrm{i} \Phi / L)$. This enables us to reach the converged results in smaller order of the polynomial expansion. We confirmed that, for the range of the parameters in the present work, the 
polynomial expansion up to the 8th order is enough to obtain the 'exact' results in the limit of the infinite order.

In the TPEMC calculations, we take the threshold values in the truncations as $10^{-4}$ for the matrix product and $10^{-5}$ for the trace operation. We confirm that the truncations with these thresholds do not change the results beyond the errors.

In the following, we calculate the magnetization at a fixed doping concentration $x$ as a function of temperature. $\left(x \equiv 1-\sum_{i}\left\langle\tilde{c}_{i}^{\dagger} \tilde{c}_{i}\right\rangle / N\right.$, where the bracket denotes the thermal average for the grand canonical ensemble.) For that purpose, we need to control the chemical potential at each temperature because the band structure changes as magnetic correlations develop in this system. ${ }^{28}$ (The case of $x=0.5$ is special because $x$ is fixed for $\mu=0$ due to the particle-hole symmetry.) Instead of this laborious procedure, we fix the chemical potential $\mu$ so that the target value of $x$ is realized at $T \sim T_{\mathrm{C}}$. This leads to $T$-dependence of $x$, however, it is small in the parameter range of interests, and does not harm the results because the magnetization is not so sensitive to the small change of $x$. We confirmed that the magnetization does not show any $x$ dependence beyond its errors for this small deviation of $x$. The values of the chemical potential are taken as $\mu=0.095,0.205$, and 0.310 for $x=0.4,0.3$, and 0.2 , respectively. The results are shown with the errorbars of $x$ which represent the small change of $x$ in the corresponding temperature range.

\section{RESULTS}

\section{A. Phase diagram in the absence of the disorder}

First, we study the pure case without the random potential, namely, $\Delta=0$. Figure 1 shows the system-size extrapolation of the ferromagnetic component of the spin structure factor, $S_{\mathrm{f}}=\sum_{i j}\left\langle\boldsymbol{S}_{i} \cdot \boldsymbol{S}_{j}\right\rangle / N$, divided by the system size $N$. All the data well scale to $N^{-2 / 3}$, which is consistent with the $\boldsymbol{k}^{2}$ dependence of the energy cutoff of magnons. ${ }^{29}$ From the extrapolated values, we obtain the magnetization in the thermodynamic limit as $M=\lim _{N \rightarrow \infty} \sqrt{S_{\mathrm{f}} / N}$.

Figure 2 summarizes the temperature dependence of the magnetization in the thermodynamic limit. The data for $x=0.5$ are taken from Ref. 10. The results are well fitted by the scaling form

$$
M \propto\left(T_{\mathrm{C}}-T\right)^{\beta},
$$

as shown by the gray curves in this figure. From these fittings, we estimate the Curie temperature $T_{\mathrm{C}}$ and the critical exponent $\beta$ for each value of $x$.

The estimates of $T_{\mathrm{C}}$ are summarized in Fig. 3 (a). The results are shown also for $x>0.5$ by using the particlehole symmetry, namely, $T_{\mathrm{C}}(x)=T_{\mathrm{C}}(1-x)$. In Fig. [3(b), the $x$ dependence of $T_{\mathrm{C}}$ is compared with the kinetic energy in the ground state (perfectly polarized state), $E_{\mathrm{K}}$,

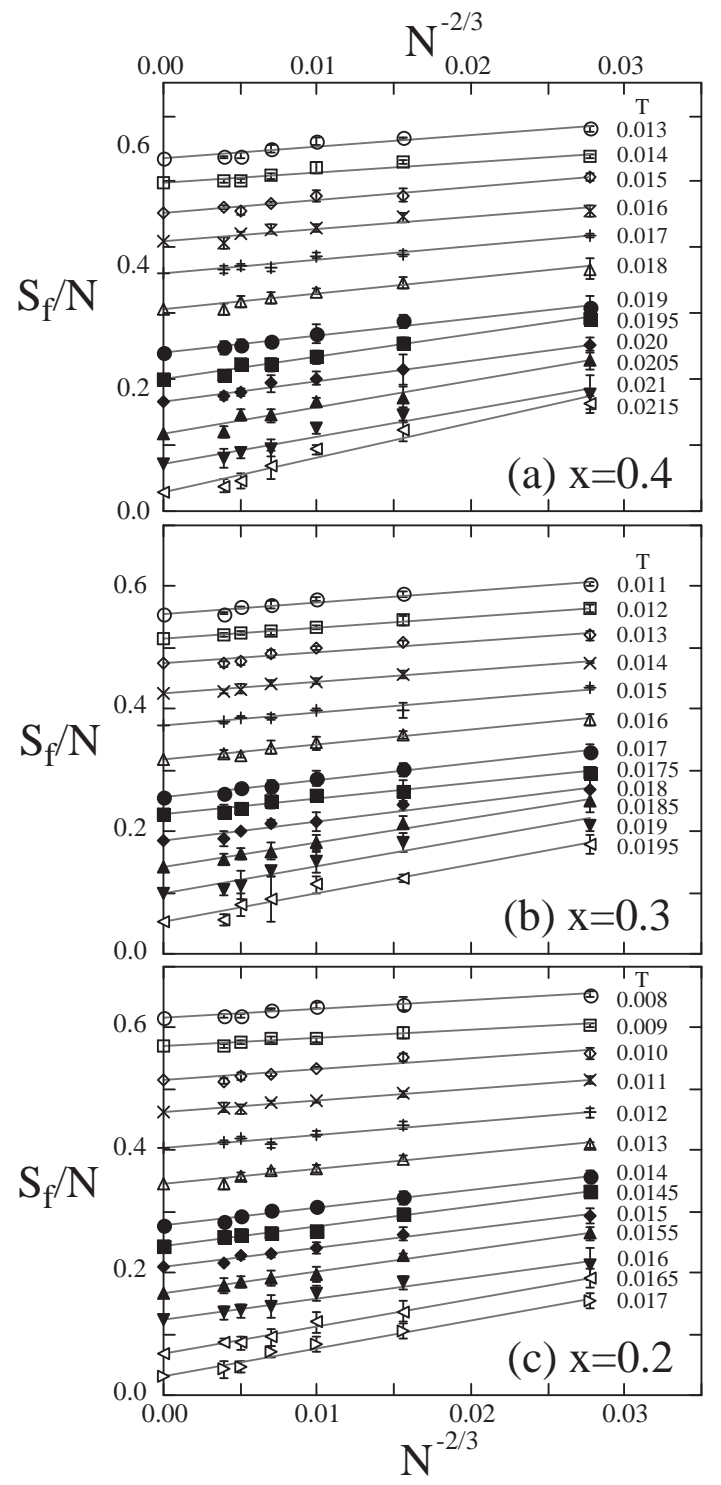

FIG. 1: System-size extrapolation of the spin structure factor in the pure case for (a) $x=0.4$, (b) $x=0.3$, and (c) $x=0.2$. Symbols at $N \rightarrow \infty$ show the extrapolated values.

and the functional form of $x(1-x)$ proposed in Ref. 31 . The data are normalized by their value at $x=0.5$ for comparison. As shown in the figure, the MC results almost scale to the kinetic energy of electrons, which confirms that the DE ferromagnetism is governed by the kinetics of electrons. From this scaling, we obtain the relation $T_{\mathrm{C}} /\left|E_{\mathrm{K}} / N\right|=0.13-0.15$. This enables us to estimate $T_{\mathrm{C}}$ approximately from the ground state quantity which is easily obtained in this DE system.

The estimates of $\beta$ by the scaling fit in Fig.2 agree with the Heisenberg exponent $\beta=0.365$ (Ref. 30) within the errors for entire range of $x$. This is consistent with the previous MC study at $x=0.5$ which has revealed that the universality class of this ferromagnetic transition belongs to that of the short-range Heisenberg model ${ }^{9.10}$ 


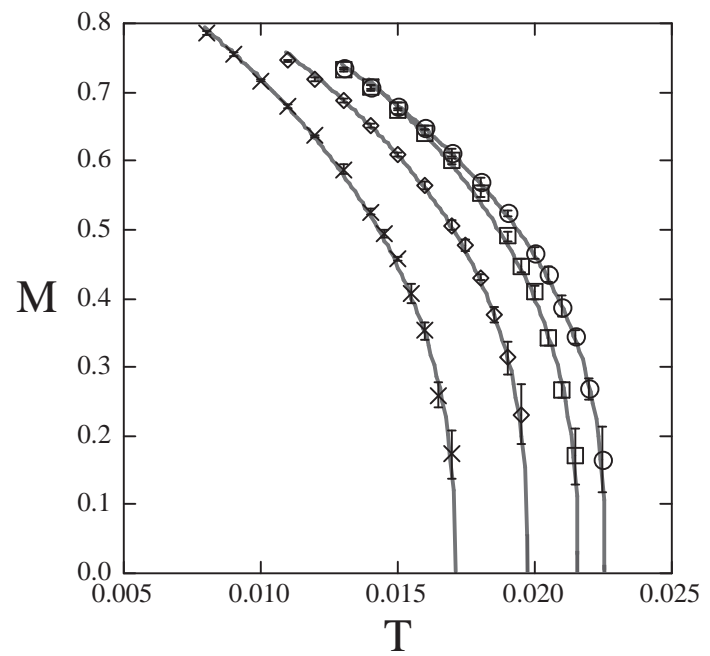

FIG. 2: Temperature dependence of the magnetization in the thermodynamic limit. Circles, squares, diamonds, and crosses represent the data for $x=0.5,0.4,0.3$, and 0.2 , respectively. The data for $x=0.5$ are from Ref. 10. The gray curves are the scaling fit by eq. (5).
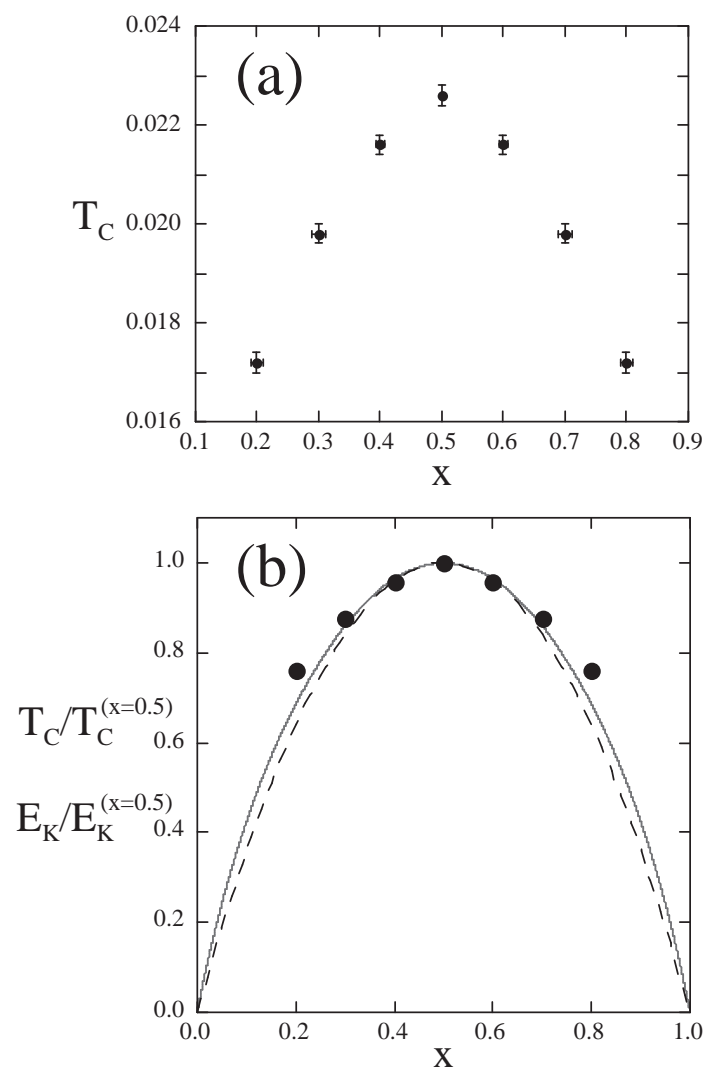

FIG. 3: (a) $x$ dependence of the Curie temperature $T_{\mathrm{C}}$. (b) Comparison of $x$ dependences of $T_{\mathrm{C}}$ (circles), the kinetic energy $E_{\mathrm{K}}$ (gray curve), and $x(1-x)$ (dashed curve). All the data are normalized by their value at $x=0.5$ for comparison.

\section{B. Disorder effect}

Next, we study the effect of the random on-site potential at $x=0.3$. Figure 4 shows the system-size extrapolation of the spin structure factor by varying the strength of the random potential $\Delta$. Even in these disordered cases, the data well scale to $N^{-2 / 3}$ as in the pure case in Fig. 1] This is consistent with recent studies for the spin excitation spectrum which predict the $\boldsymbol{k}^{2}$ magnon excitation even in the presence of disorder ${ }^{32.33 .34}$ The magnetization calculated from the extrapolated values are summarized in Fig. [5 The random potential decreases the magnetization because it reduces the kinetic energy, namely, the DE ferromagnetic interaction.

We apply the scaling of eq. (51) in this disordered case also, and obtain the estimates of $T_{\mathrm{C}}$ in Fig. [6] (a). $T_{\mathrm{C}}$ appears to scale to $\Delta^{2}$ in the weak-disorder regime. In Fig. 6 (b), we compare $\Delta$ dependence of $T_{\mathrm{C}}$ with the kinetic energy of electrons in the ground state, and find that $T_{\mathrm{C}}$ well scales to the kinetic energy. This indicates that, also in the presence of the disorder, the DE ferromagnetism is governed by the kinetics of electrons.

From the scaling fit in Fig. 5 the critical exponent $\beta$ is also estimated. The results are shown in Fig. [7 The exponent is consistent with the Heisenberg value $\beta=0.365$ (Ref. 30) even in the presence of the disorder. This indicates that the disorder is irrelevant and does not change the universality class in this transition.

\section{DISCUSSIONS}

\section{A. Comparison with $\mathrm{La}_{1-x} \mathrm{Sr}_{x} \mathrm{MnO}_{3}$}

$\mathrm{La}_{1-x} \mathrm{Sr}_{x} \mathrm{MnO}_{3}$ near $x=0.3$ has been considered to be a canonical DE system in which many aspects of the thermodynamics are successfully explained by the DE model (11) alone $\stackrel{4.9}{.}$ Here, we compare the estimates of $T_{\mathrm{C}}$ obtained in Sec. IIIA with the experimental values in this compound. Comparing the $\mathrm{MC}$ estimate $T_{\mathrm{C}}^{(\mathrm{MC})}=$ $0.0198 \mathrm{~W}$ with the experimental value $T_{\mathrm{C}}^{(\exp )}=369 \mathrm{~K}^{13}$ at $x=0.3$, we obtain the half bandwidth as $W \simeq 1.6 \mathrm{eV}$. This value is larger than the estimates by the band calculations $(W \sim 1 \mathrm{eV}) \stackrel{35,36.37}{,}$ however we note that this is the bare value in the case of $J_{\mathrm{H}}=0$ and that a large $J_{\mathrm{H}}$ generally renormalizes the bandwidth.

Figure 8 shows the comparison of $x$ dependence of $T_{\mathrm{C}}$ between theory and experiment. We normalize $T_{\mathrm{C}}^{(\mathrm{MC})}$ as well as the kinetic energy of electrons to agree with $T_{\mathrm{C}}^{(\exp )}$ at $x=0.3$. We note that, in the range of $0.15 \lesssim x \lesssim$ $0.3, T_{\mathrm{C}}^{(\exp )}$ well scales to the MC results and the kinetic energy. This agreement has been claimed in the DMFA results $\frac{17}{17}$ For $x \gtrsim 0.4, T_{\mathrm{C}}^{(\exp )}$ is suppressed and shows a deviation from this scaling. This might be due to the instability toward the A-type antiferromagnetic state or the CE-type charge-ordered state observed near $x \simeq 0.5$ 


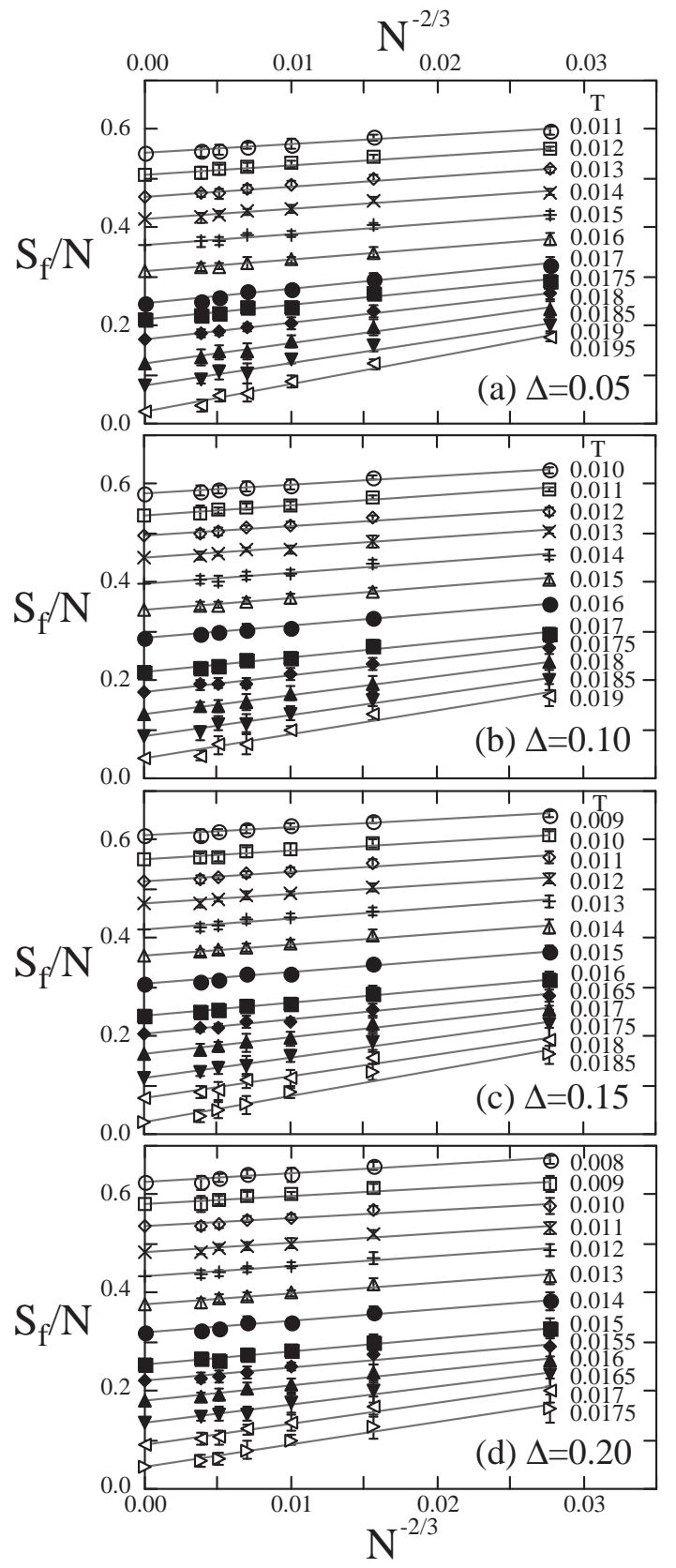

FIG. 4: System-size extrapolation of the spin structure factor at $x=0.3$ for (a) $\Delta=0.05$, (b) $\Delta=0.1$, (c) $\Delta=0.15$, and (d) $\Delta=0.2$. Symbols at $N \rightarrow \infty$ show the extrapolated values.

in many CMR manganites ${ }^{14,15,16}$ Our data suggest that if such instabilities are absent, the Curie temperature can become higher up to $\sim 420 \mathrm{~K}$.

For the critical exponents, experimental results in this compound are still controversial. The estimates are scattered from the short-range Heisenberg ones to the meanfield ones $\frac{38.39 .40 .41 .42 .43}{3}$ Our results indicate that if the DE interaction plays a dominant role in the ferromagnetic transition, the Heisenberg universality class should be observed $\frac{9,10,11,12}{2}$ Further experimental studies are de-

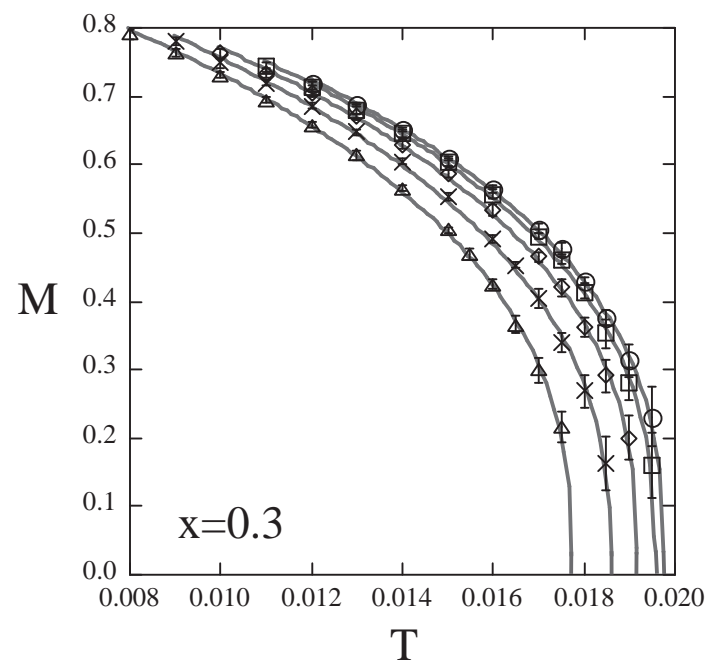

FIG. 5: Temperature dependence of the magnetization in the thermodynamic limit at $x=0.3$. Circles, squares, diamonds, crosses, and triangles represent the data for $\Delta=$ $0.0,0.05,0.1,0.15$, and 0.2 , respectively. The gray curves are the scaling fit by eq. (5).

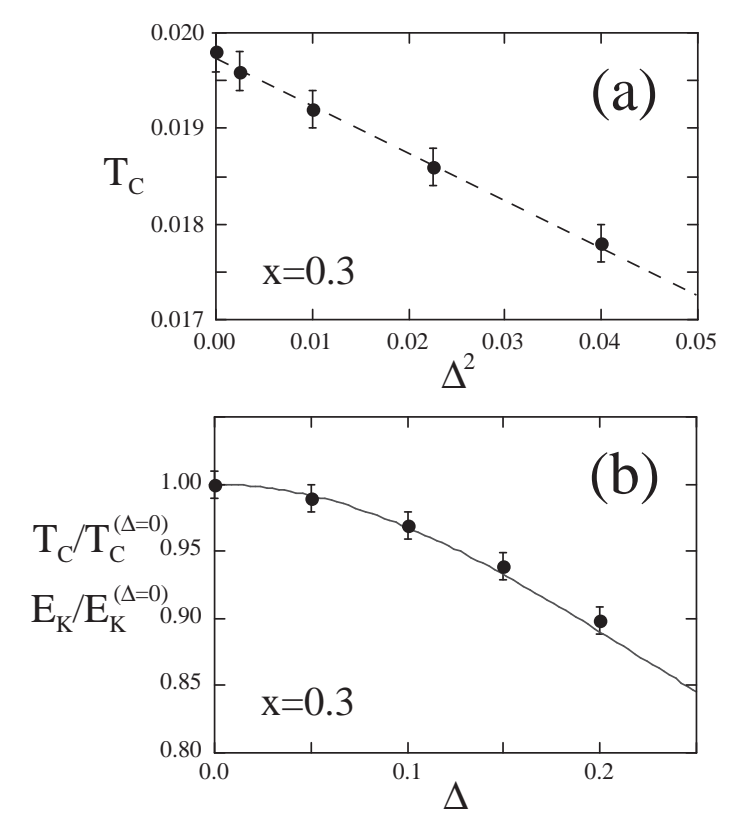

FIG. 6: (a) $T_{\mathrm{C}}$ at $x=0.3$ plotted as a function of $\Delta^{2}$. The line shows the linear fit. (b) Comparison of $\Delta$ dependences of $T_{\mathrm{C}}$ (circles) and the kinetic energy $E_{\mathrm{K}}$ (gray curve). The data are normalized by their value at $\Delta=0$ for comparison.

sired.

\section{B. Disorder effect in the $A$-site substitution}

Many experiments have indicated that the disorder is important in the $A$-site substitution in $A \mathrm{MnO}_{3}$ at a fixed doping concentration. ${ }^{23,24,25,26}$ There, the chemi- 


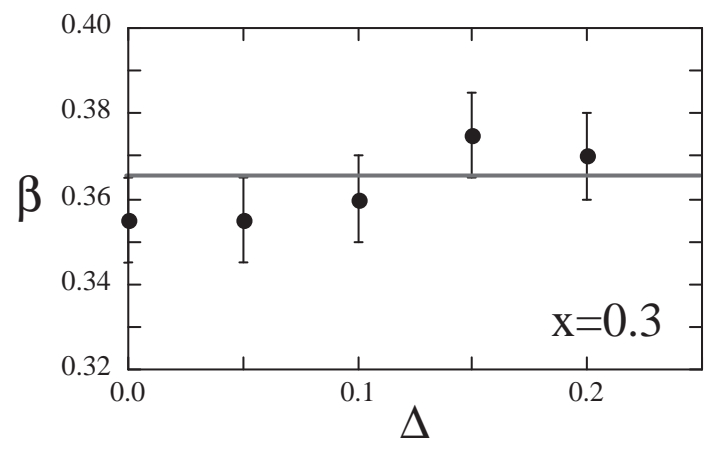

FIG. 7: $\Delta$ dependence of the critical exponent $\beta$. The horizontal line represents the Heisenberg exponent $\beta=0.365$.

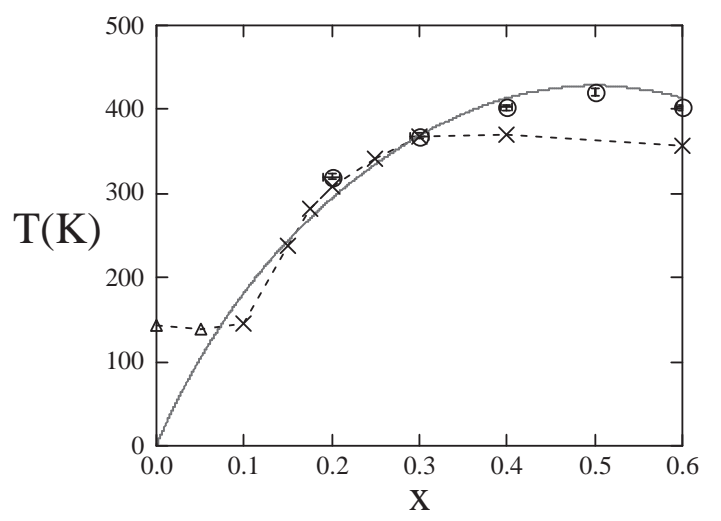

FIG. 8: Comparison between the MC results of $T_{\mathrm{C}}$ (circles) and the experimental values in $\mathrm{La}_{1-x} \mathrm{Sr}_{x} \mathrm{MnO}_{3}$ (crosses). The kinetic energy in the ground state is also shown (gray curve). All the data are normalized to agree with each other at $x=0.3$ for comparison. Triangles represent the antiferromagnetic transition temperature observed in the low $x$ regime. Experimental data are from Ref. 13.

cal disorder from the random distribution of the $A$ ions with different ionic radii is considered to disturb the electronic state in Mn-O-Mn network electrostatically and structurally. For instance, $T_{\mathrm{C}}$ decreases about $30 \%$ from $\mathrm{La}_{0.7} \mathrm{Sr}_{0.3} \mathrm{MnO}_{3}$ to $\mathrm{La}_{0.7} \mathrm{Ca}_{0.3} \mathrm{MnO}_{3}$, and this cannot be explained by the pure DE model (II) since the estimated change of the transfer integral is only about $2 \% \stackrel{23}{2}$ The MC results in Sec. IIIB shows that $T_{\mathrm{C}}$ decreases substantially by the disorder. Figure 6 (b) suggests that the $30 \%$ decrease of $T_{\mathrm{C}}$ might be achieved at $\Delta \sim 0.4-0.5$. This corresponds to $\Delta \sim 0.6-0.8 \mathrm{eV}$ if we assume $W=1.6 \mathrm{eV}$ as in Sec. IVA The estimate of the disorder strength appears to be consistent with the potential fluctuation due to the alloying effect predicted by the band calculations 36

The disorder effect has been also studied in the spin excitation spectrum recently ${ }^{32,33.34}$ The results well explain the spectral anomalies which show up in the $A$-site substituted materials $\frac{44.45 .46 .47}{T}$ This and the above observation in the decrease of $T_{\mathrm{C}}$ consistently indicate the importance of the disorder in the $A$-site substitution in
CMR manganites.

In the present MC study, we consider only the diagonal disorder of the random on-site potential. In real materials, the $A$-site disorder may affect the electronic state in other ways, for instance, as the off-diagonal disorder of the random transfer integrals. In the study of the spin excitation spectrum, it was shown that various types of disorder bring about the universal results in the spectrum 32.33 .34 We speculate that this is the case also in the thermodynamics, namely, that $T_{\mathrm{C}}$ scales to the kinetic energy and the critical exponents are for the Heisenberg universality class irrespective of the types of disorder.

For further substitution which introduces larger difference in ionic radius, experimentally, the ferromagnetic state is taken over by the charge-ordered state concomitant with the Jahn-Teller distortion ${ }^{14.15 .16}$ The phase diagram shows the multicritical behavior which indicates a strong competition between different phases ${ }^{48.49,50}$ Disorder effect on the multicritical phenomena has attracted much attentions recently ${ }^{51.52 .53}$ The emergence of the charge-ordered state suggests that, finally, another element such as the electron-phonon interaction becomes important when we approach to the multicritical point. However, we consider that, in the regime far from the multicritical point, such another element may be less important and the disorder plays a primary role.

\section{SUMMARY}

We have studied the phase diagram and the universality class of the ferromagnetic transition in the threedimensional double exchange model with and without the random potential. The truncated polynomial-expansion Monte Carlo method has been employed to calculate large size clusters without uncontrolled or biased approximations. The Curie temperature and the critical exponent have been estimated by applying the systematic finite-size scaling analysis up to $16^{3}$ site clusters. For both changes of the doping concentration and the strength of the random potential, we found that the Curie temperature $T_{\mathrm{C}}$ well scales to the kinetic energy of electrons per site in the ground state $E_{\mathrm{K}} / N$. This is a consequence of the fact that the kinetics of electrons governs the ferromagnetism in this system. From this scaling, we have obtained the approximate relation $T_{\mathrm{C}} /\left|E_{\mathrm{K}} / N\right|=0.13-0.15$, which is useful since the ground state quantity is easily calculated in the double exchange system. In both cases with and without disorder, estimates of the critical exponent are consistent with that of the Heisenberg spin model with short-range interaction. This indicates that the ferromagnetic transition in the double exchange systems belongs to the shortrange Heisenberg universality class. We have compared the results with the experimental results in Sr doping in $\mathrm{La}_{1-x} \mathrm{Sr}_{x} \mathrm{MnO}_{3}$ and in the ionic radius control by the $A$-site substitution in $A \mathrm{MnO}_{3}$. 


\section{Acknowledgment}

The authors thank H. Nakata for helpful support in developing parallel-processing systems. The computations have been performed mainly using the facilities in the AOYAMA+ project (http://www.phys.aoyama.ac.jp/ aoyama + ). This work is supported by "a Grant-in-Aid from the Ministry of Education, Culture, Sports, Science, and Technology".
${ }^{1}$ C. Zener, Phys. Rev. 82, 403 (1951).

2 P. W. Anderson and H. Hasegawa, Phys. Rev. 100, 675 (1955).

3 P. G. de Gennes, Phys. Rev. 118, 141 (1960).

${ }^{4}$ N. Furukawa, Physics of Manganites, ed. T. A. Kaplan and S. D. Mahanti (Plenum Press, New York, 1999), and references therein.

5 E. Dagotto, T. Hotta, and A. Moreo, Phys. Rep. 344, 1 (2001), and references therein.

6 Y. Motome and N. Furukawa, J. Phys. Soc. Jpn. 68, 3853 (1999).

7 N. Furukawa and Y. Motome, in preparation.

8 J. L. Alonso, L. A. Fernández, F. Guinea, V. Laliena, and V. Martín-Mayor, Nucl. Phys. B 596, 587 (2001).

9 Y. Motome and N. Furukawa, J. Phys. Soc. Jpn. 69, 3785 (2000); 70, 3186 (2001).

$10 \mathrm{Y}$. Motome and N. Furukawa, preprint (cond-mat/0304551).

11 Y. Motome and N. Furukawa, J. Phys. Soc. Jpn. 70, 1487 (2001); 70, 2802 (2001).

12 N. Furukawa and Y. Motome, Appl. Phys. A 74, 1728 (2002).

13 A. Urushibara, Y. Moritomo, T. Arima, A. Asamitsu, G. Kido, and Y. Tokura, Phys. Rev. B 51, 14103 (1995).

14 J. B. Goodenough, Phys. Rev. 100, 564 (1955).

15 H. Kuwahara, Y. Moritomo, Y. Tomioka, A. Asamitsu, M. Kasai, R. Kumai, and Y. Tokura, Phys. Rev. B 56, 9386 (1997).

16 T. Akimoto Y. Maruyama, Y. Moritomo, A. Nakamura, K. Hirota, K. Ohoyama, and M. Ohashi, Phys. Rev. B 57, 5594 (1998).

17 N. Furukawa, J. Phys. Soc. Jpn. 64, 2754 (1995).

18 R. Allub and B. Alascio, Solid State Commun. 99, 613 (1996).

19 B. M. Letfulov and J. K. Freericks, Phys. Rev. B 64, 174409 (2001).

${ }^{20}$ M. Auslender and E. Kogan, Phys. Rev. B 65, 012408 (2001).

21 E. E. Narimanov and C. M. Varma, Phys. Rev. B 65, 024429 (2002).

${ }^{22}$ Y. Motome and N. Furukawa, J. Phys. Chem. Solids 63, 1357 (2002).

23 P. G. Radaelli, G. Iannone, M. Marezio, H. Y. Hwang, S.W. Cheong, J. D. Jorgensen, and D. N. Argyriou, Phys. Rev. B 56, 8265 (1997).

${ }^{24}$ L. M. Rodriguez-Martinez and J. P. Attfield, Phys. Rev. B 54, 15622 (1996).

25 J. M. D. Coey, M. Viret, L. Ranno, and K. Ounadjela, Phys. Rev. Lett. 75, 3910 (1995).

${ }^{26}$ E. Saitoh, Y. Okimoto, Y. Tomioka, T. Katsufuji, and Y. Tokura, Phys. Rev. B 60, 10362 (1999).

27 F. F. Assaad, Phys. Rev. B 65, 115104 (2002).

28 N. Furukawa, J. Phys. Soc. Jpn. 66, 2523 (1997).
29 N. Furukawa, J. Phys. Soc. Jpn. 65, 1174 (1996).

30 J. C. Le Guillou and J. Zinn-Justin, Phys. Rev. Lett. 39, 95 (1977).

31 C. M. Varma, Phys. Rev. B 54, 7328 (1996).

32 Y. Motome and N. Furukawa, J. Phys. Soc. Jpn. 71, 1419 (2002).

33 Y. Motome and N. Furukawa, J. Phys. Soc. Jpn. 72, 472 (2003).

34 Y. Motome and N. Furukawa, in preparation.

${ }^{35}$ N. Hamada, H. Sawada, and K. Terakura, in Proc. 17th Taniguchi International Conference, eds. A. Fujimori and Y. Tokura (Springer-Verlag, Berlin, 1995).

${ }^{36}$ W. E. Pickett and D. J. Singh, Phys. Rev. B 55, 8642 (1997).

37 D. A. Papaconstantopoulos and W. E. Pickett, Phys. Rev. B 57, 12751 (1998).

38 M. C. Martin, G. Shirane, Y. Endoh, K. Hirota, Y. Moritomo, and Y. Tokura, Phys. Rev. B 53, 14285 (1996).

39 S. E. Lofland, V. Ray, P. H. Kim, S. M. Bhagat, M. A. Manheimer, and S. D. Tyagi, Phys. Rev. B 55, 2749 (1997).

${ }^{40}$ L. Vasiliu-Doloc, J. W. Lynn, Y. M. Mukovskii, A. A. Arsenov, and D. A. Shulyatev, J. Appl. Phys. 83, 7342 (1998).

41 Ch. V. Mohan, M. Seeger, H. Kronmüller, P. Murugaraj, and J. Maier, J. Mag. Mag. Mater. 183, 348 (1998).

${ }^{42}$ K. Ghosh, C. J. Lobb, R. L. Greene, S. G. Karabashev, D. A. Shulyatev, A. A. Arsenov, and Y. Mukovskii, Phys. Rev. Lett. 81, 4740 (1998).

43 A. Schwartz, M. Scheffler, and S. M. Anlage, Phys. Rev. B 61, 870 (2000).

${ }^{44}$ H. Y. Hwang, P. Dai, S.-W. Cheong, G. Aeppli, D. A. Tennant, and H. A. Mook, Phys. Rev. Lett. 80, 1316 (1998).

45 L. Vasiliu-Doloc, J. W. Lynn, A. H. Moudden, A. M. de Leon-Guevara, and A. Revcolevschi, Phys. Rev. B 58, 14913 (1998).

46 P. Dai, H. Y. Hwang, J. Zhang, J. A. Fernandez-Baca, S.W. Cheong, C. Kloc, Y. Tomioka, and Y. Tokura, Phys. Rev. B 61, 9553 (2000).

47 G. Biotteau, M. Hennion, F. Moussa, J. RodríguezCarvajal, L. Pinsard, A. Revcolevschi, Y. M. Mukovskii, and D. Shulyatev, Phys. Rev. B 64, 104421 (2001).

${ }^{48}$ Y. Tomioka and Y. Tokura, Phys. Rev. B 66, 104416 (2002).

49 D. Akahoshi, M. Uchida, Y. Tomioka, T. Arima, Y. Matsui, and Y. Tokura, to appear in Phys. Rev. Lett.

50 T. Nakajima, H. Kageyama, H. Yoshizawa, and Y. Ueda, J. Phys. Soc. Jpn. 71, 2843 (2002).

51 A. Moreo, S. Yunoki, and E. Dagotto, Science 283, 2034 (1999).

52 J. Burgy, M. Mayr, V. Martin-Mayor, A. Moreo, and E. Dagotto, Phys. Rev. Lett. 87, 277202 (2001).

${ }^{53}$ Y. Motome, N. Furukawa, and N. Nagaosa, preprint (cond-mat/0304542); preprint (cond-mat/0304543). 